\title{
"La Evaluación Psicológica en Niños. Técnicas de Screening y Diagnóstico" de Alejandro Castro Solano y Mercedes Fernández Liporace
}

\author{
Compiladores: Alejandro Castro Solano y Mercedes Fernández Liporace \\ Autores colaboradores: Luciana Becerra, Analía Brizzio, María Alejandra Carreras, Ana \\ D’Anna, Guadalupe de la Iglesia, Lucila López, María Josefina Scheinshon y Fabiana Uriel. \\ Año de edición: 2017 \\ Editorial: Paidós
}

En palabras de los compiladores, el trabajo plasmado en este libro fue guiado por una doble meta: por un lado, la actualización de los materiales de evaluación disponibles e incorporación de los nuevos hallazgos y consensos de la psicología del neurodesarrollo y de la psicopatología y; por el otro, el diseño de nuevas herramientas adecuadas a la población local de niños de 4 a 12 años. Tras una revisión detallada de los capitulos, se puede afirmar que se ha cumplido acabadamente con las metas propuestas.

Los contenidos de la obra dan cuenta de un arduo trabajo realizado tanto en el contexto de investigación - y la consecuente transferencia de tecnologíacomo en el campo aplicado.

La primera parte del libro aporta el background necesario para comprender los objetivos y pasos necesarios para realizar una apropiada y profunda evaluación psicológica en niños. Una de las principales características que diferencia a este libro y le aporta relevancia es que reúne tanto los aportes clásicos sobre la temática como los últimos avances en el campo, dando cuenta de una exhaustiva revisión de la literatura internacional. Además, contextualiza la evaluación infantil de acuerdo a las propuestas de la última versión del Manual Diagnóstico y Estadístico de los Trastornos Mentales (DSM 5, 2013).

Los aspectos centrales de la Primer Sección de la obra se relacionan con diferenciar la evaluación infantil de acuerdo con los ámbitos de aplicación siendo el educacional y clínico los más frecuentes y, en menor medida, el forense (Capitulo 1). Otro aporte fundamental de esta sección es la descripción ofrecida acerca del desarrollo neurológico y la adquisición de los hitos evolutivos en niños, brindando indicadores basados en el DSM 5 para la detección temprana de anomalías y/o alteraciones en el marco de una evaluación psicológica (Capítulo 2). Dicha información se complementa con una exhaustiva reseña de los instrumentos psicométricos de evaluación utilizados tanto a nivel internacional como local. Dicha reseña incluye tanto pruebas para la evaluación de la inteligencia como de aspectos no intelectivos (i.e. Aspectos emocionales, comportamentales y sociales) (Capítulo 3).

El profesional psicólogo verá notablemente enriquecida su labor como evaluador a partir de los instrumentos de screening y diagnóstico propuestos 
en la Segunda parte del libro. Las autoras de los capitulos pertenecientes a esta segunda sección hacen especial hincapié en la importancia de la detección temprana de posibles casos clínicos a fin de intervenir del modo más rápido posible y evitar una mayor severidad de los cuadros. Las técnicas de screening deben caracterizarse por ser altamente sensibles, poco específicas, simples y de corta duración. En este libro se propone una serie de pruebas que reúnen dichas características y que componen una nueva minibatería para el rastrillaje de aspectos madurativos de niños entre 5 y 12 años. Esta bateria actualiza la tradicional minibatería Koppitz, comúnmente empleada en la evaluación psicoeducacional. Incluye el tradicional Test de la Figura Humana (DFH) y el Test Guestáltico Visomotor de Bender que ya formaban parte de la minibatería Koppitz y que son frecuentemente usados en escuelas, hospitales y otros servicios de atención tanto privados como públicos. La novedad de esta obra es que brinda normas estadisticas locales y recientes junto con información científica actualizada para una correcta interpretación de los resultados (Capítulos 5 y 6). Además, esta novedosa minibatería propuesta se completa con un nuevo instrumento de screening diseñado por dos de los autores (Castro Solano y D'Anna). Se trata del Screening Neuropsicológico para niños UBA (SNN-UBA). Este consta de una serie de tareas sencillas (verbales y pictóricas) que evalúan diferentes funciones cognitivas. A partir de un puntaje de corte permite estimar el nivel de madurez neuropsicológica del examinado (capítulo 7).

La Segunda Sección también propone otra nueva técnica diseñada por autoras del libro (Uriel, Scheinshon, Becerra y D`Anna). La misma tiene formato de entrevista estructurada y se denomina Entrevista para la Evaluación Diagnóstica Infantil (EEDI). Se emplea en tareas de diagnóstico (ya no screening) y está formulada sobre la base de la clasificación de trastornos psicológicos en la infancia propuesta por el DSM 5. Cuenta con una primera parte que consiste en un cuestionario a padres destinado a la evaluación de los hitos del desarrollo y comportamientos adaptativos del niño y, una segunda parte, en la que se examinan indicadores de sintomatología asociada a los trastornos psicopatológicos más frecuentes en niños. Resulta sumamente apropiada su utilización en los casos que el resultado del screening propuesto por la nueva minibatería resulte positivo (Capítulo 4).

Esta obra brinda al lector todos los materiales necesarios para poder hacer uso de las pruebas propuesta en la segunda parte (protocolos, grillas de corrección, baremos y otros materiales necesarios según el test que se trate). Además, cuenta con resultados de investigaciones actuales y ejemplos de informes clínicos para orientar al profesional en la evaluación de los casos.

En sintesis, los contenidos presentados en este libro dan cuenta del exhaustivo trabajo realizado por este equipo de psicólogos y refleja la vasta experiencia tanto en tareas de investigación como de psicología aplicada con la que cuentan los compiladores y autores de esta obra. Constituye un pool esencial de herramientas teóricas y técnicas para el trabajo del psicólogo dedicado a la evaluación psicológica infantil.

\section{Maria Laura Lupano Perugini ${ }^{1}$}

\footnotetext{
${ }^{1}$ Consejo Nacional de Investigaciones Cientificas y Técnicas (CONICET), Universidad de Palermo, Argentina;
} mllupano@hotmail.com 
Lupano Perugini, M. L. / Psicodebate, 17(2), 83 - 86.

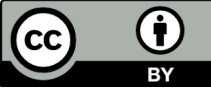

Este trabajo se encuentra bajo una Licencia Creative Commons Atribución 4.0 Internacional que permite a terceros utilizar lo publicado siempre que se dé el crédito pertinente a los autores y a Psicodebate

Psicodebate, Vol. 17, № 2, Diciembre 2017 - Mayo 2018

ISSN: 1515-2251 e-ISSN: 2451-6600 
Psicodebate, Vol. 17, N 2, Diciembre 2017 - Mayo 2018 ISSN: 1515-2251 e-ISSN: 2451-6600 